\title{
Formal Verification of Railway Timetables - Using the UPPAAL Model Checker
}

\author{
Haxthausen, Anne E.; Hede, Kristian
}

Published in:

From Software Engineering to Formal Methods and Tools, and Back

Link to article, DOI:

10.1007/978-3-030-30985-5_25

Publication date:

2019

Document Version

Early version, also known as pre-print

Link back to DTU Orbit

Citation $(A P A)$ :

Haxthausen, A. E., \& Hede, K. (2019). Formal Verification of Railway Timetables - Using the UPPAAL Model Checker. In From Software Engineering to Formal Methods and Tools, and Back (pp. 433-448). Springer. Lecture Notes in Computer Science (including subseries Lecture Notes in Artificial Intelligence and Lecture Notes in Bioinformatics) Vol. 11865 LNCS https://doi.org/10.1007/978-3-030-30985-5_25

\section{General rights}

Copyright and moral rights for the publications made accessible in the public portal are retained by the authors and/or other copyright owners and it is a condition of accessing publications that users recognise and abide by the legal requirements associated with these rights.

- Users may download and print one copy of any publication from the public portal for the purpose of private study or research.

- You may not further distribute the material or use it for any profit-making activity or commercial gain

- You may freely distribute the URL identifying the publication in the public portal 


\title{
Formal Verification of Railway Timetables - Using the UPPAAL Model Checker
}

\author{
Anne E. Haxthausen and Kristian Hede \\ DTU Compute, Technical University of Denmark, Kongens Lyngby, Denmark. \\ aeha@dtu.dk and krhede@gmail.com
}

\begin{abstract}
This paper considers the challenge of validating railway timetables and investigates how formal methods can be used for that. The paper presents a re-configurable, formal model which can be configured with a timetable for a railway network, properties of that network, and various timetabling parameters (such as station and line capacities, headways, and minimum running times) constraining the allowed train behaviour. The formal model describes the system behaviour of trains driving according to the given railway timetable. Model checking can then be used to check that driving according to the timetable does not lead to illegal system states. The method has successfully been applied to a real world case study: a time table for 12 trains at Nærumbanen in Denmark.
\end{abstract}

Keywords: formal methods · model checking - UPPAAL · railways · timetables

\section{Introduction}

This paper considers the challenge of validating time tables in the railway domain.

Background. As timetables specify how the trains should run in the railway network, they have a great influence on the railway operations. Therefore, for any railway operator, it is of very high priority that the timetables are feasible as well as robust against delays. However, the process of creating train timetables [6] is very complicated and goes through many steps taking various wishes, requirements and scheduling constraints into account. Traditionally, time tables are created in a stepwise manner, where schedules are manually adjusted until all constraints are met. ${ }^{1}$ When a timetable has been created in this way, it should be verified that it is feasible and satisfies all the many stated requirements and scheduling constraints. For instance, it should be checked that at any time the number of trains waiting at a station does not exceed the station capacity and that the minimal headways between trains are satisfied. Tools for automated checking of all such requirements are highly needed. Today there are

\footnotetext{
${ }^{1}$ Research in optimisation models and techniques for generating optimal timetables have been done [6], but these are very rarely used by the railway operators.
} 
some timetabling tools [10] like RailSys and TPS on the market which can be used for managing timetables and estimating the effect of train delays. The tools typically have functionality for displaying time tables graphically (showing the planned train positions as a function of time). In order to verify a timetable, one can inspect such graphs and manually check whether the requirements are fulfilled. Although this is very useful, it would be desirable to have a tool that could automatically check all requirements.

Contribution. In this paper we propose a fully automated method for verifying timetables. This method is formal and based on model checking. The idea is as follows: To check a timetable for a given railway network, one should create a real-time model simulating how trains move around in the network, from station to station over time, according to the timetable. During the simulation, a number of required properties of timetables should be checked. If any of these fail, the system should go into an error state. Then one can model check that all trains reach their final destination without ending in an error state. We have explored this idea using UPPAAL[3], which is an integrated tool environment for modelling, validation and verification of real-time systems modelled as networks of timed automata. We choose to use UPPAAL as our system is a real-time system and the UPPAAL symbolic model checker has shown to very be effective. The UPPAAL model is re-configurable: It can be configured with a timetable and a railway network description including various scheduling parameters (such as station and line capacities, headways, and minimum running times) constraining the allowed train behaviour. The verification method has successfully been applied to a real world case study: a time table for 12 trains at Nærumbanen Local Railway in Denmark.

To our best knowledge it is a novelty to verify railway timetables by model checking.

Related work. Over the last decade formal methods have been widely used for developing railway systems, cf., the surveys given in $[2,5]$. According to these surveys, formal methods have mostly been applied to railway interlocking systems $^{2}$ (as e.g. in $[11,8,7,12,1]$ ) and other control system components, but less for railway operations, where formal methods have primarily been used for capacity analyses (as e.g. in [4]).

Paper overview. First, in Sect. 2 and Sect. 3, short, informal introductions to the UPPAAL modelling language and to the domain of railway timetables are given, respectively. Then Sect. 4 presents a formal UPPAAL model of trains driving according to a given railway time table, whereupon, in Sect. 5 , it is described how the verification of model instances is done. Finally, Sect. 6 gives a conclusion and states ideas for future work.

\footnotetext{
${ }^{2}$ An interlocking system is a signalling system component responsible for the safe routing of trains through a railway network.
} 


\section{The UPPAAL Modelling Language}

This section gives an ultra short, informal introduction to the major UPPAAL modelling language constructs used in this paper. The reader is assumed familiar with the theory of timed automata. For more details, especially on semantics of the concurrency construct, the reader should consult [3].

In UPPAAL a system is modelled as a network of parallel timed automata (called processes) which are finite-state machines extended with time in the form of real-valued clocks which progress synchronously and with data variables of simple data types (bounded integers, arrays, etc.).

The specification of a system model consists of (1) templates for timed automata, (2) declarations of clocks, data variables, constants, channels, and functions which can be used in the templates, and (3) a system declaration which is a parallel composition of processes which are instances of the templates. The processes can communicate asynchronously via shared variables or synchronously via channels.

A timed automaton consists of locations and edges.

In the UPPAAL graphical representation of timed automata, locations are shown as circles and may have a name shown in red colour. The initial location is shown by double circles. A location may be committed (shown as a circle with a $\mathrm{C}$ inside). If a process is in a committed location, the time must not pass and the next system transition must involve an edge from a committed location. A location may be labelled with an invariant (shown in purple colour) which is Boolean expression over variables and clocks. The process can stay in that location and let the time pass as long as the invariant is satisfied, but when the invariant becomes false, it must leave the location.

In the graphical representation, edges are shown as arrows. An edge may be labelled with (1) a guard (shown in green colour) which is Boolean expression over variables and clocks determining when the edge is enabled and can be fired, (2) updates of variables and clocks (shown in blue colour) that should be executed when the edge is fired, and (3) synchronisations of the form $c$ ? or $c$ ! over a channel $c$ (shown in light blue colour). (An edge labelled with $c$ ! in one process may synchronise with an edge labelled with $c$ ? in another process provided that both edges are enabled.)

\section{Domain Description}

This section presents the definitions of basic terms of timetabling, as used in this paper, and requirements to timetables.

\subsection{Basic concepts and terms}

Railway networks. A railway network consists of stations and open lines between stations. A station can be a passenger station where people embark and disembark trains, or a technical station where e.g. trains can overtake each other. 
An open line is a collection of tracks between two stations. In this paper it is assumed that each open line is either a single track or is a double track (has two tracks). ${ }^{3}$ For double track lines it is assumed that there is one track dedicated to each driving direction.

Railway timetables. A railway timetable for a given railway network is a collection of individual train timetables/schedules for train journeys in the network. Each train timetable has a unique (train) identifier and a list of stations at which the train should stop, along with the arrival and departure times at those stops.

A train is said to drive according to its timetable, if it arrives at and departs from the stations in its timetable at the stated arrival and departure times.

Parameters in timetabling. The requirements to timetables are expressed in terms of a number of scheduling parameters described below. These depend among others on the track layout, train properties, and signalling system properties. It is out of the scope to explain how these parameters are determined - for an explanation of that, see e.g. [9]. Often some time supplements are added to / included in the minimum times mentioned below to make the timetable robust against small train delays.

The capacity of a station is the maximal number of trains that are allowed to be at the station at the same time. (In this paper, for simplicity, we will assume that if a station has capacity $n$, then it consists of $n$ parallel tracks, each track having capacity for one train. Furthermore, we will assume that each station track is connected to each open line track.) The minimum dwell time of a train at a station is the minimum time it must wait at the station, such that there is time to open and close doors and let people enter and exit the train.

The capacity of an open line is the maximal number of trains that are allowed to be on each of its tracks at the same time. An open line also has a minimum running time, i.e. the time it must at least take for a train to drive between the two stations connected by the open line. Furthermore, an open line has a minimum headway, i.e. the minimum time that must pass between two following trains both entering the same track or both leaving the same track. Stations can also have different kinds of minimum headways between two trains entering/leaving the stations (depending on the signalling system). In this paper we just illustrate how this can be done for a minimum headway between two trains entering the station.

\subsection{Requirements}

For a timetable to be valid, a number of requirements must hold when the trains drive according to the timetables. Below we state examples of typical requirements, but these might differ for different railways.

\footnotetext{
${ }^{3}$ In Denmark this is the case for most railway networks, with only very few exceptions.
} 
no overtaking: Trains must not overtake another train on the same track of an open line (as this is physically not possible and would prevent the plan from being executable.)

no opposing trains: Single track open lines must not be utilised in both directions simultaneously (as that would lead to deadlocks preventing the plan from being executable.)

open line running times: Trains must satisfy the minimum running times of the open lines.

open line capacities: The capacity of open lines must never be exceeded.

open line headways: The headway times of open lines must be respected.

station dwell times: Trains must satisfy the dwell times at the stations.

station capacities: The capacity of stations must never be exceeded.

station headways: The headway times of stations must be respected.

It is seen how the two first requirements concern internal inconsistencies in a given timetable (in this case conflicting train schedules) ${ }^{4}$, while the remaining ones concern the timetabling parameters identified above.

\section{UPPAAL Model}

This section presents the UPPAAL model created for verifying timetables. The model can be found here: http://www2 . compute.dtu.dk/ aeha/RobustRails/ data/timetabling/uppaal-models/. The model has been designed to be reconfigurable, so that it can be re-used for a whole class of railway networks and timetables, without having to change the templates of the model, but only constant data representing the railway network and the timetables.

\subsection{Overview}

The model consists of the following three parts:

1. Global declarations of (1) the configuration data (constants) defining a railway network layout and timetabling parameters and a timetable for that network, (2) clocks, (3) variables, (4) types, and (5) functions.

2. Two templates: Train and Initialiser. A process instance of the Train template represents a single train, running according to one of the timetables in the global declarations. The Initialiser template is used to initialise the system.

3. The system declaration, which creates a single Initialiser process and a Train process for each of the trains in the timetable.

The following sections describe these parts, except the types and functions.

${ }^{4}$ Note that the interlocking system has the responsibility to ensure that collisions would not happen in such cases. 


\subsection{Railway network data}

The railway network data (describing the network layout and the timetabling parameters) are represented by a collection of constants as shown in Listing 1.1, where the constants are configured for the network of Nærumbanen in Denmark which has 9 stations connected one by one by single track open lines. The first constants give the number of stations, the number of open lines, and the station ids. The stationTable is an array with one entry for each station giving the capacity (capacity) and minimum headway (HWT) for that station. The openLineTable is an array with one entry for each open line (represented as a pair of the two stations it is connecting) stating the kind of line (false means single track and true means double track) and giving the minimum running time (MRT), the capacity (capacity), and the minimum headway (HWT) for that open line.

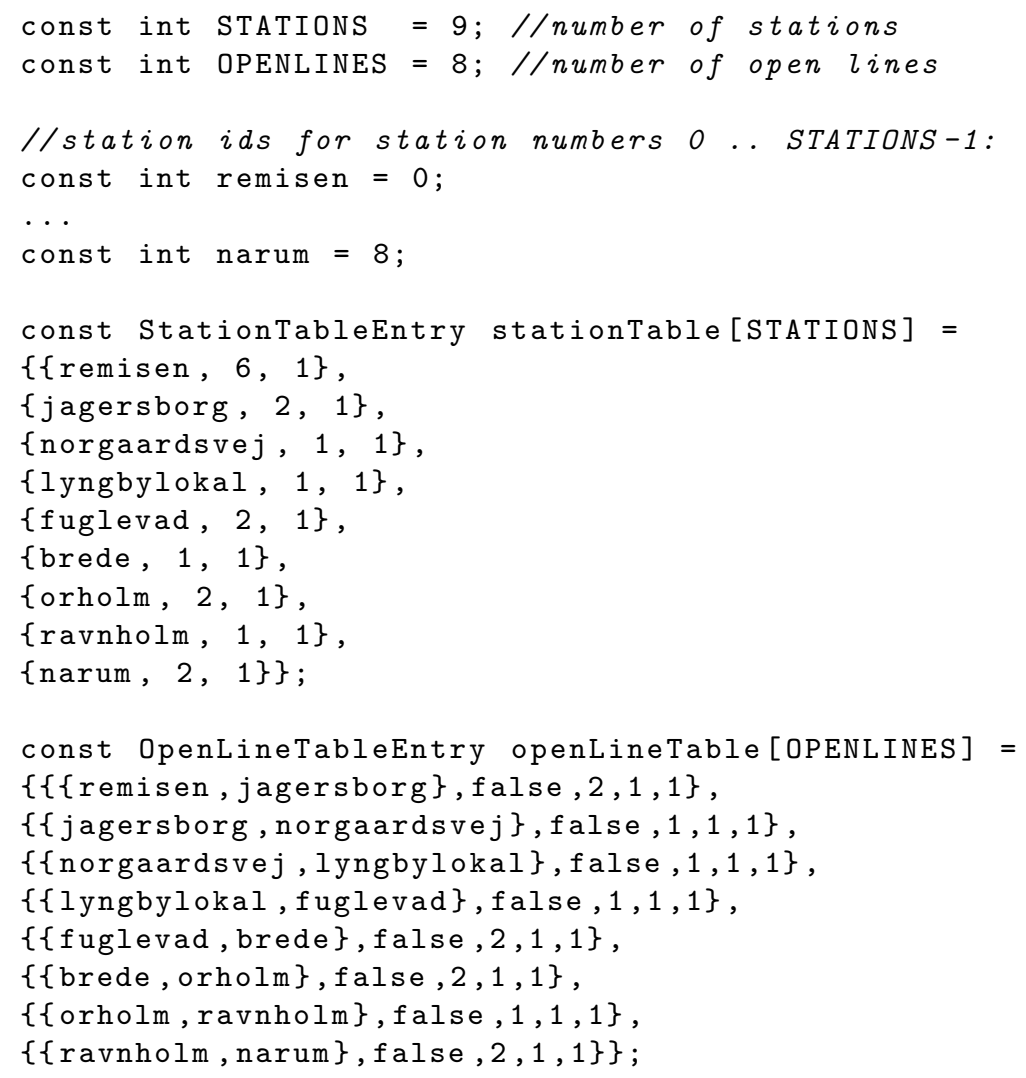

Listing 1.1. Example of network data for Nærumbanen.

\subsection{Timetable data}

The railway timetable is represented by a collection of constants as shown in Listing 1.2, where the constants are configured for an extract of the timetable of 
Narumbanen in Denmark. stops is an array of timetables - one for each train, and each timetable is an array of stop entries. Each stop entry gives the station id (StationID), the arrival time (AT), the departure time (DT), and the minimum dwell time ${ }^{5}$ (DWT) for a stop. For the shown train timetables the minimum dwell time has been chosen to be zero for stations where the trains need not to stop.

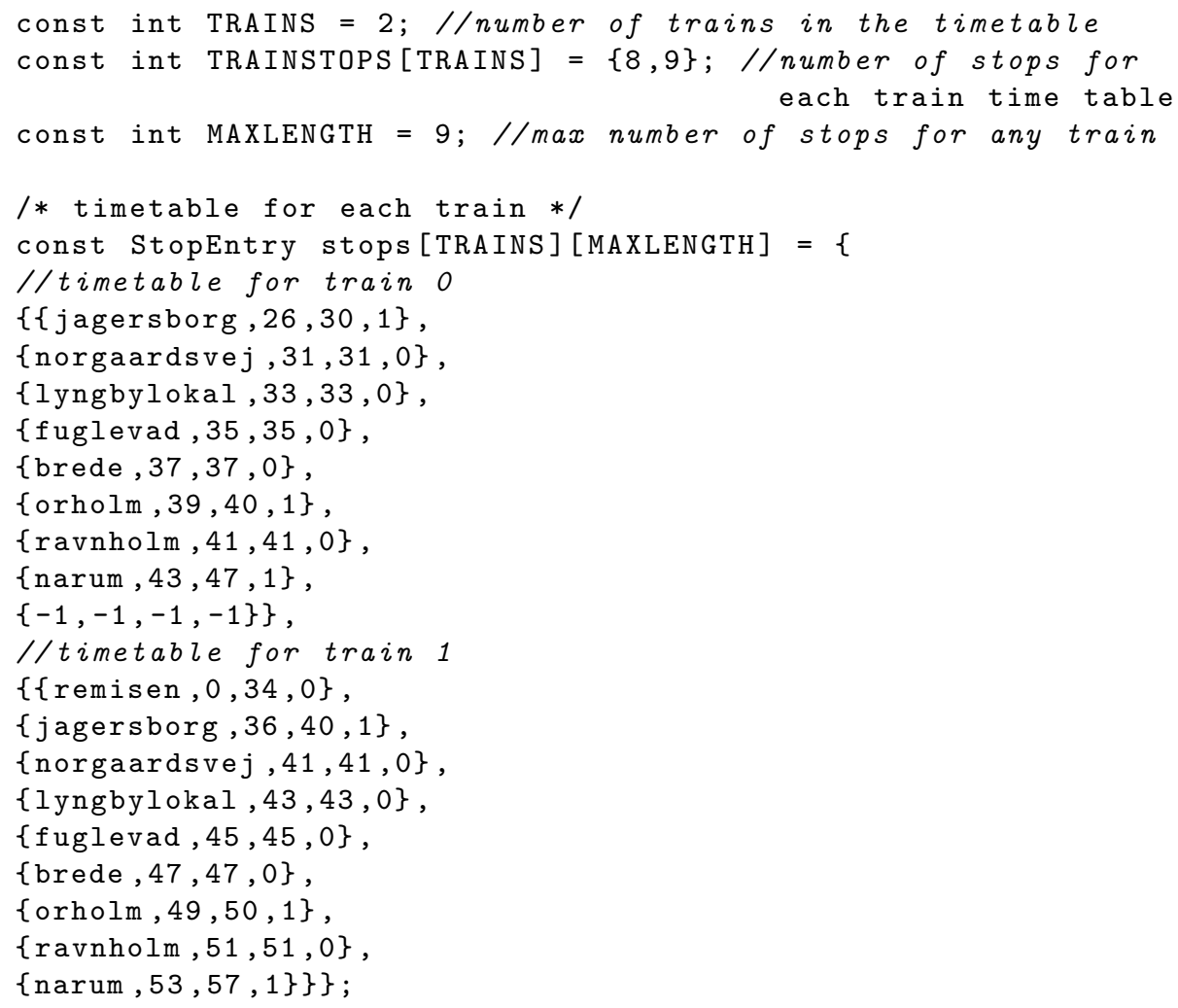

Listing 1.2. Example of a timetable for Nærumbanen.

\subsection{Clocks}

To express time constraints in the model, a number of clocks are introduced in addition to the system clock time:

clock TrainClock[TRAINS];

clock openLineLastEntered [OPENLINES] [2] ;

clock openLineLastExit [OPENLINES] [2];

clock stationLastEntered[STATIONS];

For each train id, TrainClock [id] is used to record how long time the train has been in its current location. For each open line number o (which is an index in the

5 To allow for different minimum dwell times at the same station for different trains, this scheduling parameter has been placed here and not in stationTable. 
openLineTable) and driving direction $d \in 0$.. 1, openLineLastEntered [o] [d] and openLineLastExit [o] [d] records the time elapsed since a train last time entered $o$ and exited $o$ in direction d, respectively. Direction 0 is used for the direction towards the first station found in the open line entry openLinetable [o], and direction 1 is used for the other direction. For each station id s, stationLastEntered [s] records the time elapsed since a train last time entered $\mathbf{s}$.

\subsection{Variables}

To express requirement checks in the model, a number of variables are introduced:

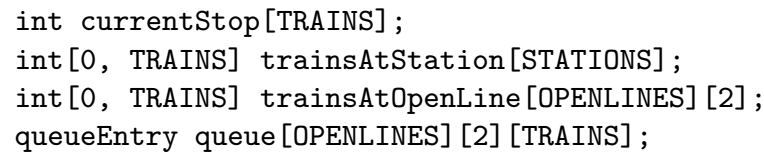

For each train id $t$, currentStop [t] stores the station id of the station at which the train is currently waiting or towards which it is currently moving (when it is on an open line). For each station s, trainsAtStation [s] stores the number of trains currently waiting at $\mathbf{s}$. For each open line number $o$ and direction $\mathrm{d} \in 0$ .. 1, trainsAtOpenLine [o] [d] stores the number of trains currently driving on $\circ$ in direction $d$. For each open line number $o$ and direction $d$, queue [o] [d] is a queue (an array) of entries for trains currently present on o in direction d. Each entry contains the id of a train and the time it is planned to leave the open line. The trains appear in the order they entered o, with the head in queue [o] [d] [0] .

\subsection{The Initialiser template}

The Initialiser template is shown in Figure 1. The initial location is commit-

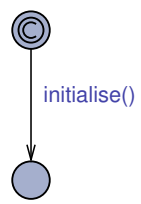

Fig. 1. The Initialiser Template.

ted making the outgoing edge of that location, the first to be fired in the system - without any delay. This edge invokes a function to initialise global variables and clocks. 


\subsection{The Train template}

The Train template has a single parameter, id, which can be instantiated with the identifier of a train from the timetable. The resulting train process simulates the behaviour of the train driving perfectly according to its timetable in stops [id] without any deviations. During the simulation, the timetable is checked to satisfy the requirements stated in Sect. 3.2.

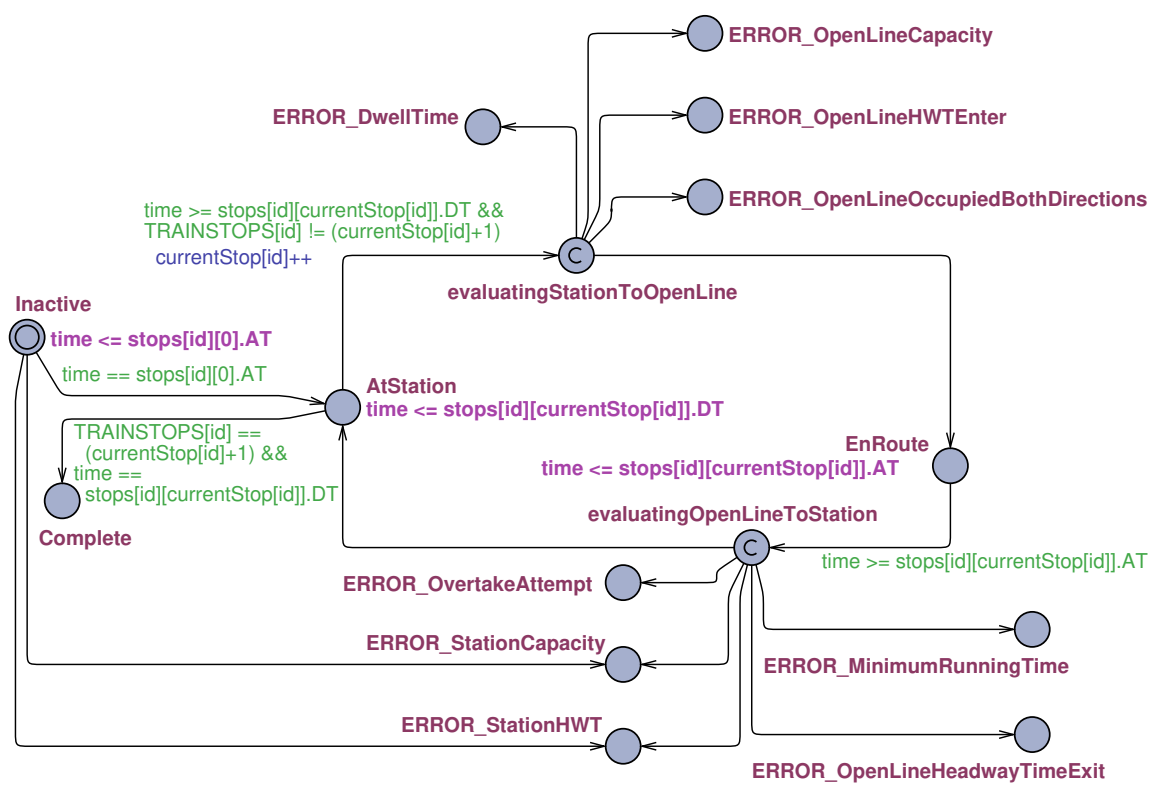

Fig. 2. The Train Template only showing state invariants and edge labels expressing conditions ensuring that the train drives according to its time table.

Figure 2 shows the template with all its locations and edges, but for readability only with those state invariants and edge labels that enforce the train to drive according to its time table. Later, the remaining edge labels will be explained.

As it can be seen, a train has the following locations:

- Inactive which is the initial location.

- AtStation which reflects that at the current time the train is waiting at a station according to the timetable.

- EnRoute which reflects that at the current time the train is driving on the open line between two consecutive stations in its timetable.

- evaluatingStationToOpenLine which is a committed, intermediate location between AtStation and EnRoute. It is an auxiliary location (not existing in a real system) which is only used for checking that neither leaving the current station nor entering the next open line would break a requirement. 
- evaluatingOpenLineToStation which is a committed, intermediate location between EnRoute and AtStation. It is an auxiliary location (not existing in a real system) which is only used for checking that neither leaving the current open line nor entering the next station would break a requirement.

- An error location for each possible error (breaking a requirement). For instance, if the open line capacity is exceeded at time $t$, the error state ERROR_OpenLineCapacity will be reached at that time.

- Complete which is a location that is reached if and when the train has successfully run through its whole timetable and reached its destination without any errors have been detected. If all trains reach this location, the complete railway timetable has successfully been validated.

Figure 2 shows those state invariants and edge labels that enforce the train to drive according to its time table: A train starts in the Inactive location. At the start time of its timetable (i.e. the arrival time stops [id] [0] . AT of its first stop) it will enter the AtStation location modelling that it is at the first stop of its timetable. Whenever the AtStation location is entered, if the train has reached its final stop (TRAINSTOPS [id] == currentStop [id] +1 ) and final time of the timetable, it will immediately go to the Complete location. Otherwise, it must leave the AtStation location and enter the evaluatingStationToOpenLine location at its departure time stops[id] [currentStop[id]].DT for its current station. During that transition the currentStop [id] counter is incremented by one.

In the evaluatingStationToOpenLine location it is checked whether some requirements are broken. If that is the case, the system goes into an error location, and otherwise it goes to the EnRoute location.

Similarly, whenever the train is in the EnRoute location (i.e. is driving on the open line towards the next station in its route), it must leave the location and enter the evaluatingOpenLineToStation location at the arrival time stops [id] [currentStop [id]]. AT for the train's next station.

In the evaluatingOpenLineToStation location it is checked whether some requirements are broken. If that is the case, the system goes into an error location, and otherwise it goes to the AtStation location.

Figure 3 shows the full model with all edge labels included. The additional guards and variable/clock updates are used to express the checks for the requirements stated in Sect. 3.2. On each edge from the evaluatingStationToOpenLine and evaluatingOpenLineToStation locations to an error state for a certain kind of error, there is a guard expressing that this error is found. The guard of the edge from the evaluatingStationToOpenLine/evaluatingOpenLineToStation location to the EnRoute/AtStation location is a conjunction of the negated error conditions in the guards of each of the edges leading to an error location from the evaluatingStationToOpenLine/evaluatingOpenLineToStation location. The guards are expressed in terms of the clocks and variables declared in Sections Sect. 4.4 and Sect. 4.5. These clocks and variables are updated when a train is entering a station or an open line. 


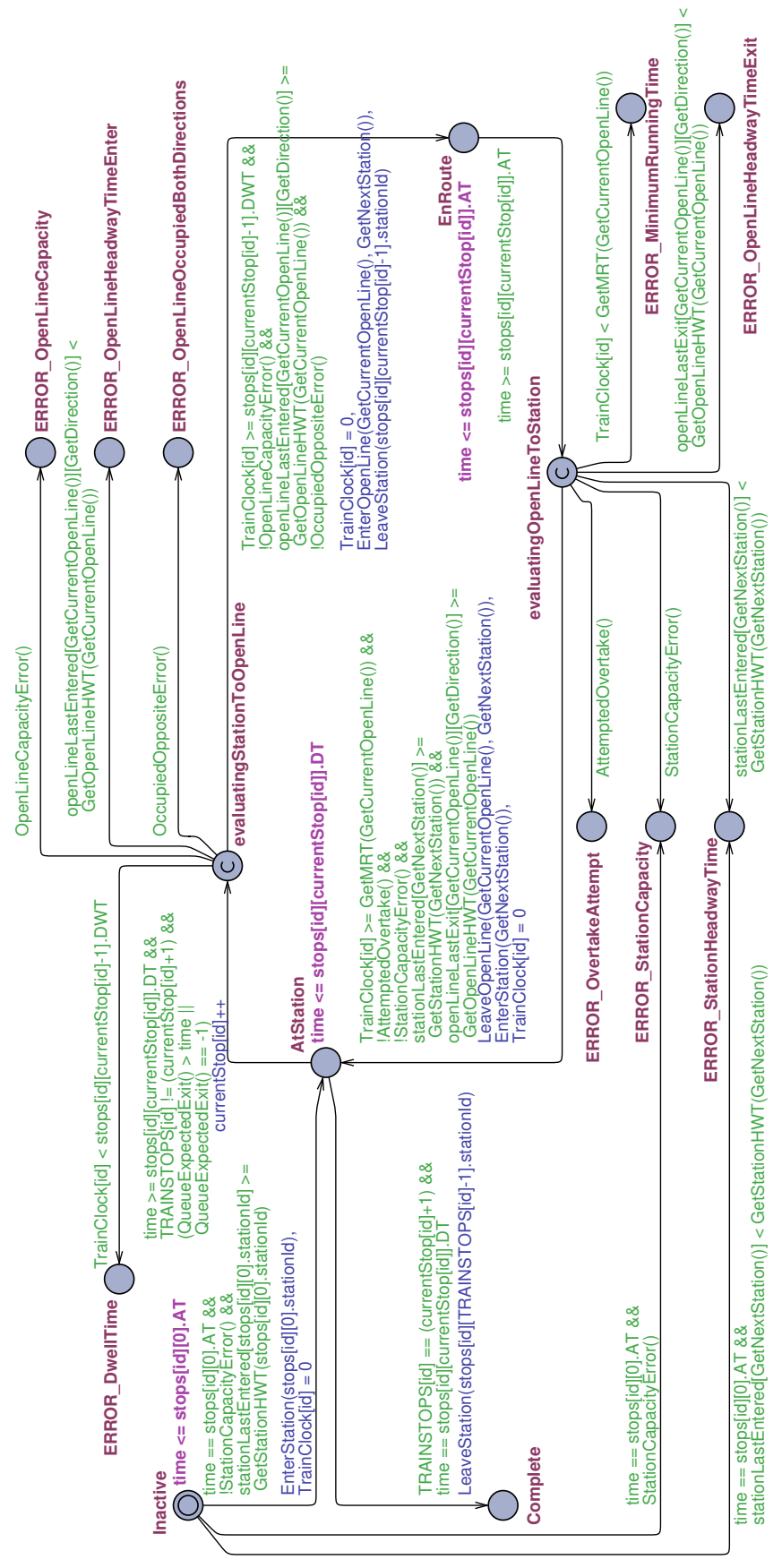

Fig. 3. The complete Train Template. 
Below we will give an overall idea of how the updates and checks for the various requirements are done. ${ }^{6}$

The openline running times requirement: In order to be able to measure how long time a train has been driving on an open line, the TrainClock [id] clock is reset to 0 each time the train enters the EnRoute location. Then, the following condition, when evaluated in the evaluatingOpenLineToStation location (i.e. when the train leaves the open line), expresses that the train has at least spent the minimum running time of the current open line in the EnRoute location:

TrainClock[id] >= GetMRT(GetCurrentOpenLine())

where GetCurrentOpenLine is an auxiliary function which returns the current open line o and GetMRT(o) returns the minimum running time of $o$ as stated in the openLineTable: openLineTable [o] .MRT.

The dwell times requirement is checked similarly using TrainClock [id].

The station headways requirement is checked in a similar way using the stationLastEntered [s] clock which is reset (by an EnterStation(s) function call) each time a train enters station $\mathbf{s}$.

The open line headways requirements for entering and leaving an open line o are checked in a similar way using the openLineLastEntered [o] [d] and the openLineLastExit [o] [d] clocks, respectively. These clocks are reset to 0 (by EnterOpenLine (o, s) and LeaveOpenLine (o, s) function calls, respectively, where $\mathbf{s}$ is the next station/stop) each time some train enters $\circ$ and leaves $\circ$ in direction $d^{7}$ (towards $s$ ), respectively.

The station capacity requirement: In order to be able to count the number of trains present on a station s, the trainsAtStation [s] variable is incremented by 1 (by an EnterStation(s) function call) each time some train enters $\mathbf{s}$ and is decremented by 1 (by a LeaveStation( $\mathbf{s}$ ) function call) each time some train leaves $\mathbf{s}$. Before the train is entering a new station $\mathbf{s}$, the following condition is used to check that the capacity of that station will not be exceeded, if the train enters the station:

trainsAtStation [s] < GetStationCapacity(s)

where GetStationCapacity(s) returns the station capacity of $s$ as stated in the stationTable: stationTable [s] .capacity

The open line capacity requirement is checked in a similar way for an open line o using the trainsAtOpenLine [o] [d] variables which are incremented/decremented (by an EnterOpenLine (o, s)/LeaveOpenLine (o, s) function call) each time a train enters/leaves o in direction d (towards $s$ ).

The no opposing trains requirement is checked before a train is entering a single track open line $o$ in direction $d$ by the condition

trainsAtOpenLine [o] [d'] $=0$

where $d^{\prime}$ is the opposite direction of $d$.

\footnotetext{
${ }^{6}$ There is no space to show and explain the many auxiliary functions used in the edge labels to implement that.

$7 \mathrm{~d}$ can be found from $\mathrm{s}$ as explained in Sect. 4.4 .
} 
The no overtaking requirement is checked using the queue [o] [d] variables (introduced in Sect. 4.5). Each time some train enters/leaves an open line o in direction $d$ towards the next station $s$, a train entry for that train (keeping its name and planed arrival time at next station) is pushed/popped to/from the queue in queue [o] [d] (by an EnterOpenLine (o, s)/LeaveOpenLine (o, s) function call). When a train is leaving its current open line o in direction d, the following condition is used to check that the first train in the queue queue [o] [d] is the train it-self which means that it has not overtaken any train:

GetQueueFirstTrain(o, d) = id where GetQueueFirstTrain(o, d) returns the train id in queue [o] [d] [0].

A concurrency issue: If one train $t_{1}$ enters an open line $\circ$ at the same time $t$ (according to the timetable) as another train $t_{2}$ leaves the same open line according to the timetable (both driving in the same direction $\mathrm{d}$ ), the interleaving semantics of UPPAAL would lead to two different traces sequencing the two events: one in which $t_{1}$ enters first and $t_{2}$ leaves afterwards, and one in which the order is opposite. If we assume that there are no other trains on the line, the value of trainsAtOpenLine [o] [d] will at time $t$ in the first trace first be 2 and then 1 , while in the other trace it will be 1 in both states. If the capacity of the line is 1 , then the first trace will give rise to a false line capacity error. Therefore, we should ensure that only the second trace is considered. In order to ensure this, the following time constraint was added to the guard of the edge from the AtStation location to the evaluatingStationToOpenLine location:

QueueExpectedExit() $>$ time

where QueueExpectedExit() returns the expected exit time of the train in the head of the queue in queue [o] [d]: queue [o] [d] [0] .expectedExit. This would mean, that a train can only enter the open line at time time, if the next train that will leave the same open line in the same direction will do that later on. Hence, for the example above, trace 1 would not anymore be possible.

Note that we do not have the same concurrency issue for stations, since if one train enters the station at the same time as another leaves it, there should be space for both of them at the same time, so both traces should be considered.

\subsection{System declaration}

The system declaration

system Train, Initialiser;

creates a single Initialiser process and a Train process Train(id) for each train identifier id, id $=0, \ldots$, TRAIN-1.

\section{Verification}

This section explains how a railway timetable can be verified by model checking a system model instantiated with data for that timetable against some properties. 


\subsection{Properties}

To verify that a railway timetable is valid, it should be checked that eventually all the trains have reached their destination as expressed by the property

A $<$ forall(i: t_id) Train(i).Complete

If this property is true, it in particular follows that no error states have been reached and therefore all the requirements stated in Sect. 3.2 are fulfilled for trains driving according to the timetable.

However, if the property fails, one can't from the property itself conclude what went wrong. In such a case, if one wishes to know which kind of errors were encountered and which trains did not reach their destination, one can instead check

A [] forall(i: t_id) not Train(i).ERROR_S

for each error location $E R R O R_{-} S$, and

Train(i). Inactive --> Train(i). Complete

and for each train $i$.

\subsection{Verification results}

For an extended version of the model created in Sect. 3.2 for Nærumbanen (now with 12 trains), we used the symbolic model checker of UPPAAL to check the properties above. All properties were successfully verified as shown by green lamps in Figure 4.

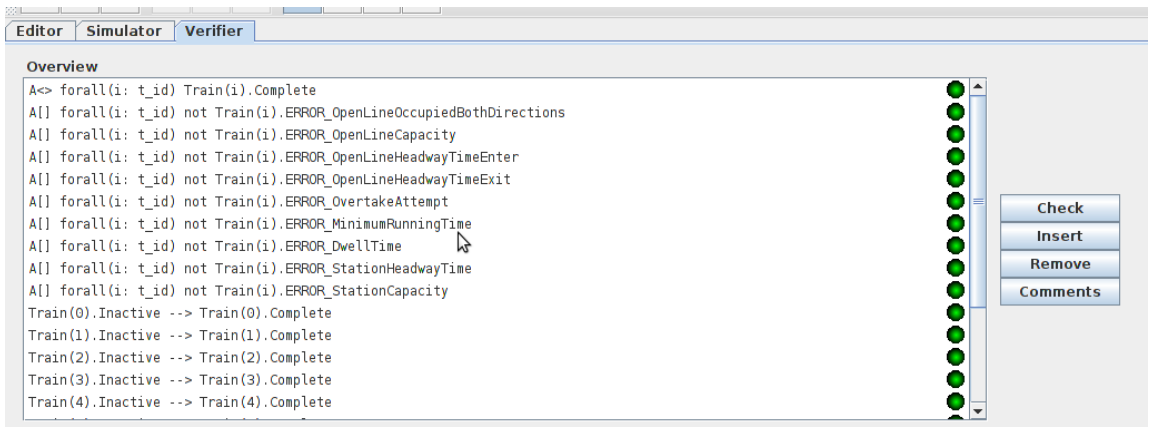

Fig. 4. Verification results for the timetable for Nærumbanen in Denmark.

Verification $^{8}$ using the A<> forall (i: t_id) Train(i). Complete property took less than a second. The alternative (more informative) verification of all the detailed properties (for individual errors and individual trains) took 5 - 6 seconds.

${ }^{8}$ The experiments were done with an Intel(R) Core(TM) i5-5200U processor at 2.2 $\mathrm{GHz}$ with 12 GB RAM. 
We also made examples with illegal timetables to test that the model checker would be able to catch the different kinds of errors. For instance, we changed the capacity of orholm station from 2 to 1 , such that the station capacity would be exceeded when two trains should pass each other at the station.

Once an error has been detected, it is possible to identify how, where and when it occurred by choosing the 'Some' option of the Diagnostic Trace. Then the failing query should be chosen and verified by itself, resulting in a diagnostic trace, which will provide the entire diagnostic trace up until the state of the error.

\section{Conclusion and Future Plans}

This paper has shown how railway timetables can be formally verified to be feasible (executable) and satisfy a number of scheduling constraints. The UPPAAL model checker was used for that purpose. To our knowledge, it is the first time that model checking has been used for that. The approach is quite promising as it was applied successfully to a real-world case study for which the timetable for 12 trains on a railway with 9 stations was successfully verified in less than a second. In future work, it would be interesting to apply the method to larger examples.

It should be straight forward to extend the model to allow more than two tracks on open lines and take more details of station topologies into account (e.g. the number of platforms and which open line tracks they are connected to) and include scheduled track occupation information for trains in their timetables. Furthermore, it could be interesting to investigate how model checking could be used for investigating the effects of train delays.

We have also investigated how model checking can be used to generate timetables and we plan to describe those results in a future paper.

\section{Acknowledgements}

This paper is dedicated to Professor Stefania Gnesi on the occasion of her 65th birthday. The first author have had the great pleasure of meeting Stefania Gnesi at many occasions and would like to thank her for inspiration, discussions, and collaboration, especially in European Technical Working Group on Formal Methods in Railway Control.

The research was partially funded by the RobustRailS project (2012-17) granted by Innovation Fund Denmark under grant agreement 0603-00483B. The authors are grateful to the organisers of the Festschrift Symposium for giving the opportunity to prepare this paper. We would like to thank the anonymous reviewers for their comments, and we would like to thank Alex Landex for discussions and sharing of his deep knowledge about railway timetabling while he was employed as a researcher in railway operations at DTU Transport. 


\section{References}

1. Banci, M., Fantechi, A., Gnesi, S.: Some Experiences on Formal Specification of Railway Interlocking Systems Using Statecharts. In: TRain Workshop at SEFM (2005)

2. Basile, D., ter Beek, M.H., Fantechi, A., Gnesi, S., Mazzanti, F., Piattino, A., Trentini, D., Ferrari, A.: On the industrial uptake of formal methods in the railway domain - A survey with stakeholders. In: Furia, C.A., Winter, K. (eds.) Integrated Formal Methods. pp. 20-29. Springer International Publishing (2018)

3. Behrmann, G., David, A., Larsen, K.G.: A Tutorial on UPPAAL. In: Bernardo, M., Corradini, F. (eds.) Formal Methods for the Design of Real-Time Systems: International School on Formal Methods for the Design of Computer, Communication, and Software Systems, Revised Lectures, pp. 200-236. Springer Berlin Heidelberg, Berlin, Heidelberg (2004)

4. Di Giandomenico, F., Fantechi, A., Gnesi, S., Itria, M.L.: Stochastic model-based analysis of railway operation to support traffic planning. In: Gorbenko, A., Romanovsky, A., Kharchenko, V. (eds.) Software Engineering for Resilient Systems. pp. 184-198. Springer Berlin Heidelberg, Berlin, Heidelberg (2013)

5. Fantechi, A.: Twenty-Five Years of Formal Methods and Railways: What Next? In: Counsell, S., Núñez, M. (eds.) Software Engineering and Formal Methods. Lecture Notes in Computer Science, vol. 8368, pp. 167-183. Springer (2014)

6. Hansen, I.A., Pachl, J.: Railway Timetable \& Traffic: Analysis, Modelling, Simulation. Eurailpress (2008)

7. James, P., Moller, F., Nguyen, H.N., Roggenbach, M., Schneider, S., Treharne, H.: Techniques for modelling and verifying railway interlockings. International Journal on Software Tools for Technology Transfer 16(6), 685-711 (2014)

8. Khan, U., Ahmad, J., Saeed, T., Hayat, S.: Real Time Modeling of Interlocking Control System of Rawalpindi Cantt Train Yard. In: 2015 13th International Conference on Frontiers of Information Technology (FIT). pp. 347-352 (2015). https://doi.org/10.1109/FIT.2015.28

9. Landex, A., Kaas, A., Hansen, S.: Railway Operation. Tech. rep., Technical University of Denmark, Centre for Traffic and Transport (2006)

10. Schittenhelm, B., Landex, A.: Jernbanesimuleringsværktøjer i Danmark (Eng.: Railway Simulation Tools in Denmark). In: Aalborg Trafikdage 2008, available from www.trafikdage.dk/papers_2008/praesentationer/bernd_schittenhelm_ 158.pdf (2008), in Danish

11. Vu, L.H., Haxthausen, A.E., Peleska, J.: Formal Modelling and Verification of Interlocking Systems Featuring Sequential Release. Science of Computer Programming 133, Part 2, 91-115 (2017). https://doi.org/http://dx.doi.org/10.1016/j.scico.2016.05.010, //www.sciencedirect.com/science/article/pii/S0167642316300570, http://dx.doi.org/10.1016/j.scico.2016.05.010

12. Winter, K.: Symbolic Model Checking for Interlocking Systems. In: Flammini, F. (ed.) Railway safety, reliability, and security: technologies and systems engineering. IGI Global (2012) 